\title{
Receptive Anal Intercourse and HIV Infection
}

\author{
Gilbert R. Lavoieㄹ, John F. Fisher ${ }^{2}$ \\ ${ }^{1}$ Medical Institute for Sexual Health, Austin, Texas, USA \\ ${ }^{2}$ Department of Infectious Disease, Medical College of Georgia, Augusta University, Augusta, Georgia, USA \\ Email: glavoie@massmed.org
}

How to cite this paper: Lavoie, G.R. and Fisher, J.F. (2017) Receptive Anal Intercourse and HIV Infection. World Journal of AIDS, 7, 269-278.

https://doi.org/10.4236/wja.2017.74023

Received: October 20, 2017

Accepted: December 26, 2017

Published: December 29, 2017

Copyright $\odot 2017$ by author and Scientific Research Publishing Inc. This work is licensed under the Creative Commons Attribution International License (CC BY 4.0).

http://creativecommons.org/licenses/by/4.0/

cc) (i) Open Access

\begin{abstract}
Objective: Inform the public that receptive anal intercourse (RAI) is a key transmission route of the human immunodeficiency virus (HIV) epidemic in the United States. Method: The role of receptive anal intercourse (RAI) in this epidemic will be examined using the following approach: 1) Risk comparison of HIV transmission via RAI to the other major routes of transmission. 2) HIV transmission risks of RAI using some of the present risk reduction techniques. 3) HIV infection via anal intercourse among male youth. 4) HIV infection via anal intercourse among women. Results: Of the major transmission routes of HIV, receptive anal intercourse has the highest transmission risk for acquiring HIV infection. RAI is 2 times the risk of needle-sharing during injection drug use (IDU) and 17 times the risk of receptive vaginal intercourse. The estimated per act probability of acquiring HIV from an infected source by the exposure route of RAI is high in most circumstances: 1) Condoms alone only partially reduce the high risk of RAI. With the addition of pre-exposure prophylaxis (PrEP), the risk is further reduced. 2) When one or both partners are infected with a sexually transmitted disease (STD), even with condom use, the risks are very high. With the addition of PrEP the risks are reduced, but RAI still carries significant risks. 3) With exposure to acute HIV (high viral load) the risks of RAI are very high and remain significant even with condom use and PrEP. Anal intercourse often begins in adolescence; both genders are at risk. Conclusion: Public knowledge of the high transmission risks of receptive anal intercourse may likely result in a downward trend of new HIV infections and contribute to ending the epidemic.
\end{abstract}

\section{Keywords}

HIV Transmission Risk, Receptive Anal Intercourse, Anal Intercourse, STD, Condom Use

\section{Introduction}

Essential to the prevention of human immunodeficiency virus (HIV) is the pub- 
lic knowing that receptive anal intercourse (RAI) is a key transmission route of the HIV epidemic in the United States (U.S.). Receptive anal intercourse (RAI) carries the highest risk for the transmission of HIV compared to all other major HIV transmission behaviors including needle-sharing during injection drug use (IDU) [1] [2]. It is well established that anal intercourse (AI) is the main route of HIV transmission among men who have sex with men (MSM) [3] [4] [5]. Heterosexuals who engage in AI are also at risk. Experimentation with AI often begins in adolescence and with increasing age, becomes more common among young adults of both genders [6] [7]. As a result, among the young there is a high number of HIV infections occurring yearly [8] [9] [10] in spite of continuing progress in understanding the pathogenesis and treatment of HIV infection.

As stated in The White House Office of National AIDS Policy of July 2015, "Educating young people about HIV before they begin engaging in behaviors that place them at risk for HIV infection should be a priority" [11]. Accordingly, the purpose of this study is to compile and organize available data from the medical literature and the Centers for Disease Control and Prevention (CDC) on the high risk of HIV transmission via RAI so as to place into perspective the significance of this high-risk behavior.

\section{Background}

Since the beginning of the U.S. HIV epidemic in the early 1980s, more cases of HIV infection have been attributed to the transmission route of AI than to any other route of transmission. With years of informing the public about HIV infection, promoting the use of condoms, introducing highly active antiretroviral therapy (HAART), introducing pre-exposure prophylaxis (PrEP), and implementing the National HIV/AIDS Strategy for the U.S. [11], the annual incidence of HIV infections has stabilized and is slowly decreasing among a number of categories of transmission. From 2010 through 2014 in the U.S., the yearly total number of new HIV infections has decreased from 43,978 to 40,234. Nevertheless, the number of new cases attributed to men who have sex with men (MSM) has remained stable [12].

In 2015 the total number of new HIV cases in the U.S. was $39,513(81 \%$ among males and 19\% among females). Seventy percent of all new HIV infections occurred among MSM (including MSM and IDU). Heterosexual contact accounted for $24 \%$ of the HIV infections [12]. In this heterosexual category, vaginal intercourse (VI) and AI are the major transmission routes [13]. Six percent of the HIV infections were acquired by equipment sharing (e.g., needle-sharing) during injection drug use (IDU) [12].

HIV infection predominantly occurs among young persons. HIV infection is now considered a chronic disease, but it still carries for the young a lifetime requirement of medication and an increased morbidity and mortality, especially for the many who are not retained in care [14] [15]. From 2010 through 2014, the all-cause mortality among HIV-infected persons with stage 3 (AIDS) in the 
U.S. was 66,984. Of this total, 40,316 (60\%) were under the age of 55 years [16]. Among newly infected persons in 2015 in the U.S., adolescents and young adults aged 13 - 24 years accounted for more than one out of five new HIV infections (22\%), and $66 \%$ of all the newly infected persons were under the age of 40 years [12].

\section{Methods}

The role of receptive anal intercourse (RAI) in this epidemic will be examined using the following approach:

1) Risk comparison of HIV transmission via RAI to the other major routes of transmission.

2) HIV transmission risks of RAI using some of the present risk reduction techniques.

3) HIV infection via AI among male youth.

4) HIV infection via AI among women.

5) Only published data are included.

\section{Results}

1) Risk comparison of HIV transmission via receptive anal intercourse (RAI) to the other major routes of transmission.

The three major routes of HIV transmission are: AI, VI, and IDU. In 2005 the CDC published an estimated per act probability of acquiring HIV from an infected source by exposure route based on studies from published medical journals [17]. The risk per 10,000 exposures to an infected source for the three major routes of transmission was: RAI: 50 ; insertive $\mathrm{AI}$ : 6.5 ; receptive penile VI: 10 ; insertive penile VI: 5; needle-sharing during IDU: 67 [17]. Comparing exposure risks, the estimated risk of unprotected RAI was 5 times that of unprotected receptive VI and 8 times the risk of insertive AI. Rather strikingly, the risk of RAI approached that of needle-sharing during IDU.

In June 2014, the CDC updated the risks of HIV transmission by routes of exposure based upon a systematic review published in the journal, AIDS [2]. According to this investigation the estimated per act probability of acquiring HIV from an infected source by exposure route follows: (risk per 10,000 exposures to an infected source) RAI: 138; insertive AI: 11 ; receptive penile VI: 8; insertive penile VI: 4; and needle-sharing during IDU: 63 [1] [2]. These results now show that RAI has the highest exposure risk for the transmission of HIV (Figure 1 [1] [2]), 17 times that of unprotected receptive VI, 13 times the risk of insertive $\mathrm{AI}$, and twice the risk of needle-sharing during IDU.

2) HIV transmission risks of receptive anal intercourse (RAI) using some of the present risk reduction techniques.

Correct and consistent condom use reduces the risk of acquiring HIV with an HIV-positive partner by an estimated 63\% for insertive AI and an estimated $72 \%$ for RAI [18] [19]. Pre-exposure prophylaxis (PrEP), a daily medication used by 


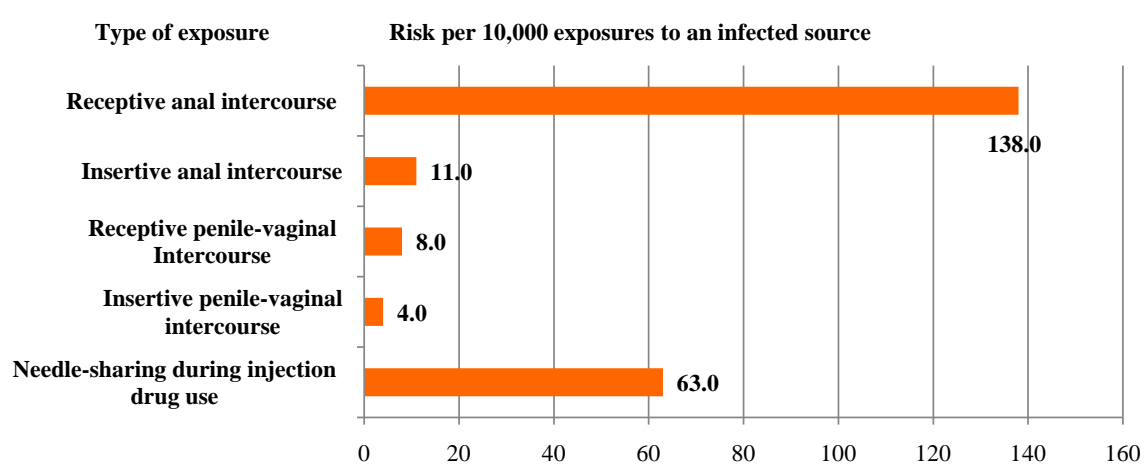

Figure 1. Estimated per-act probability of acquiring HIV from an infected source by exposure act. Derived from: Centers for Disease Control and Prevention. HIV Risk Behaviors. 2014; http://www.cdc.gov/hiv/policies/law/risk.html.

HIV-negative persons at high risk for acquiring HIV, can reduce the risk of acquiring HIV by $90 \%$. Using PrEP plus a condom provides even greater protection [18]. As to the long-term consistent use of condoms, it was also noted that although men who have sex with men (MSM) had received sustained behavioral interventions for $\geq 1$ year, the reported percentage of consistent condom use during AI was low (16.4\%). This may explain, in part, why the high rates of HIV infection among MSM have persisted [19].

HIV transmission risks may be estimated under many circumstances using the CDC's HIV Risk Reduction Tool [20]. Table 1 [20] derived from the Tool, illustrates very high risks for HIV transmission via RAI as compared to insertive $\mathrm{AI}$ and receptive VI in both asymptomatic (chronic) HIV infection and in the acute retroviral syndrome (new infection, high viral load). Specifically, when the inserter is HIV-positive and the receptor is HIV-negative, the estimated per act probability of acquiring HIV via RAI with a condom is 39 (risk per 10,000 exposures to an infected source) [20]. This is a higher risk than insertive AI without a condom (risk: 11). RAI with a condom plus PrEP reduces the risk to 3 per 10,000 exposures. However, if the insertive partner has acute HIV infection (high viral load) the risk for RAI with a condom is 280 , and with a condom and PrEP it is 22 [20]. Thus, despite risk reduction with a condom and PrEP, the RAI HIV-negative partner remains at risk. Again, that risk is higher than having insertive AI without a condom.

It has been long known that ulcerative and non-ulcerative sexually transmitted diseases (STDs) increase the risk of HIV transmission [21] [22]. However, it has been more recently realized that there is a very high risk of HIV transmission via RAI among persons infected with an STD, even with condom use. If either partner has an STD, or if both have an STD, the risk can escalate to extreme risk especially if exposed to acute HIV. Without a condom, the risks range from (chronic) 356 to (acute) 6840. With a condom the risks range from (chronic) 100 to (acute) 1915. Again, these risks can be reduced with the added option of PrEP. However, the risks still range from (chronic) 8 to (acute) 153. A risk of 
Table 1. ${ }^{* \star}$ Estimated Per-Act Probability of Acquiring HIV from an Infected Source, by Exposure Act. Risk per 10,000 exposures to an infected source.

\begin{tabular}{|c|c|c|c|}
\hline & $\begin{array}{l}\text { Receptive } \\
\text { Anal Sex }\end{array}$ & $\begin{array}{l}\text { Insertive } \\
\text { Anal sex }\end{array}$ & $\begin{array}{l}\text { Receptive } \\
\text { Vaginal Sex }\end{array}$ \\
\hline \multicolumn{4}{|l|}{ HIV (chronic-untreated) } \\
\hline No condom & 138 & 11 & 8 \\
\hline one with $\mathrm{STD}^{* * *}$ & $356^{*}$ & $28^{*}$ & 21 \\
\hline both with STD & 944 & 75 & 55 \\
\hline Condom & 39 & 4 & 2 \\
\hline one with STD & 100 & 11 & 4 \\
\hline both with STD & 264 & 28 & 11 \\
\hline Condom and PrEP & 3 & $<0.5$ & $<0.5$ \\
\hline one with STD & 8 & 1 & $<0.5$ \\
\hline both with STD & 21 & 2 & 1 \\
\hline \multicolumn{4}{|l|}{$\begin{array}{c}\text { Acute HIV } \\
\text { (high viral load-untreated) }\end{array}$} \\
\hline No condom & 1001 & 80 & 58 \\
\hline one with STD & $2581^{\star}$ & $206^{*}$ & $150^{*}$ \\
\hline both with STD & 6840 & 545 & 397 \\
\hline Condom & 280 & 30 & 12 \\
\hline one with STD & $723^{*}$ & $76^{*}$ & $30^{*}$ \\
\hline both with STD & 1915 & 202 & 79 \\
\hline Condom and PrEP & 22 & 2 & 1 \\
\hline one with STD & $58^{\star}$ & 6 & 3 \\
\hline both with STD & 153 & 16 & 8 \\
\hline
\end{tabular}

${ }^{*}$ Could be slightly higher depending on which partner (inserter/receptor) has the STD. ${ }^{*}$ Derived from

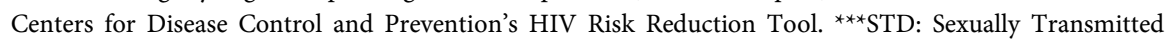
Disease. ${ }^{* * *}$ PrEP: Pre-exposure prophylaxis.

8 per 10,000 exposures is the same risk that is carried by unprotected receptive VI (Table 1 [20]).

The high risks of RAI shown on the Table explain why the CDC states that since condoms are not $100 \%$ effective, persons are advised to consider using more than one risk reduction option (for example, a condom plus PrEP) at the same time during AI. The CDC also advises choosing less risky behaviors than AI [18].

If the HIV infected, insertive partner is being treated and is taking antiretroviral therapy (ART) correctly, the risk via RAI transmission can be reduced by 96\% [18]. The estimated probability per act via RAI is reduced from 138 to 6 (risk per 10,000 exposures to an infected source) [20]. Treated individuals who 
use condoms further reduce the risk of HIV transmission to 2. If their receptive partner is on PrEP, the risk is further reduced to $<0.5$. For further details on risk information on treated HIV infection, see the CDC's HIV Risk Reduction Tool [20]. Diagnosis and treatment is an ongoing part of the U.S. HIV prevention strategy [11]. Its efficacy in prevention depends on linkage to HIV medical care, retention in care, and suppression of viral load with ART [14] [15] [16].

3) HIV infection via AI among male youth.

HIV infection among MSM youth has been rising among all the major races. In the U.S. from 2001 through 2006 the estimated number of HIV/AIDS cases attributed to MSM in the age group 13 - 24 years by race and year of diagnosis is illustrated (Figure 2 [8]). From 2006 to 2009, the number of HIV infections attributed to MSM aged 13 - 24 years increased by more than 50\% [9]. From 2010 through 2014 HIV infections attributed to MSM among adolescents 13 - 19 years decreased, but among young adults 20 - 24 years it increased [10].

The behavior of heterosexual AI among males often starts in adolescence. A 2002 National Survey of Family Growth (NSFG) study of adolescent males documented that by age 15, 4.6\% had had insertive AI with the opposite gender, and by age 24 the percentage had risen to $34 \%$ (Figure 3 [6]).

4) HIV infection and AI among women

It has been known for many years that receptive anal intercourse (RAI) also places women at high risk for HIV infection. The European Study Group on Heterosexual Transmission of HIV, published in 1992, demonstrated that unprotected RAI was five-fold more likely to transmit HIV than unprotected receptive VI [13] [17]. Now RAI is known to be 17 times more likely to transmit HIV than receptive VI [1] [2].

Again, the behavior of heterosexual AI among females often starts in adolescence. A 2002 National Survey of Family Growth (NSFG) study of adolescent and young adult females documented that by age $15,2.4 \%$ had had RAI with the

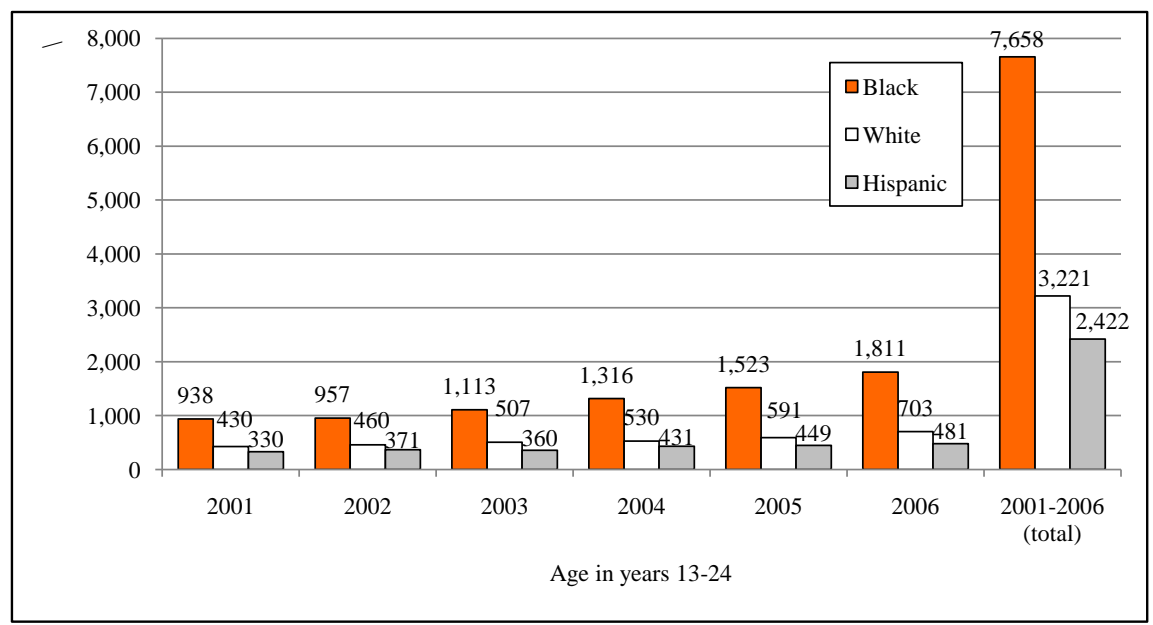

Figure 2. Estimated number of HIV/AIDS diagnoses attributed to MSM in the age group 13 - 24 years by race/ethnicity and year of diagnosis, in 33 states of the U.S., 2001-2006. CDC. Morbidity and Mortality Report, July 27, 2008/Vol. 57/No. 25, Table. 


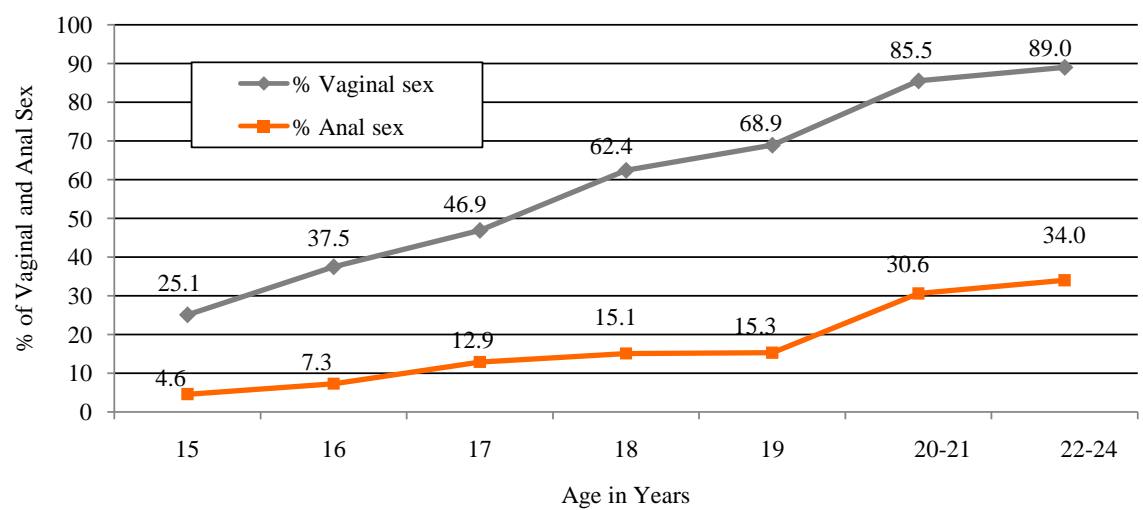

Figure 3. National Survey of Family Growth U.S. 2002. Heterosexual Vaginal and Anal Intercourse Reported by Young Men. Mosher WD, Chandra A, Jones J. Sexual behavior and selected health measures: men and women 15 - 44 years of age, United States, 2002. Adv Data. 2005 (362): 1-55.

opposite gender, and by age 24 the percentage had risen to $32.1 \%$ (Figure 4 [6]).

Among adolescent and adult women, the behaviors of heterosexual VI and AI play major roles in the transmission of HIV infection when there is a higher prevalence of HIV infection in a community. In 2014 in the U.S., the prevalence rate of HIV infection per 100,000 population for blacks (1025.2) was nearly three times the rate for Hispanics (358.3) and nearly seven times the rate for whites (151.7) [12]. In 2015, the annual HIV diagnosis rate per 100,000 population for black women (26.2) was nearly five times the rate for Hispanic women (5.3) and greater than 16 times the rate for white women (1.6) [12]. Therefore, the higher prevalence rates of HIV and the apparent common practice of RAI nationally, could explain, in part, why the HIV diagnosis rates are high for Hispanic women and higher for black women. However, the significance of the contribution of RAI in the transmission of HIV infection remains undetermined since national VI and AI data on women who have acquired HIV infection (heterosexual category) are not available.

\section{Discussion}

Overall, receptive anal intercourse (RAI) is a key transmitter of HIV infection. The complicated epidemiology of the role of RAI in HIV infections has become better understood with advances in risk-factor analysis. For example, in 2005 the risk of transmission of HIV via receptive anal intercourse (RAI) was believed to have been lower than that of needle-sharing during IDU, and five times that of receptive VI. RAI is now known to be twice the risk of needle-sharing during IDU and 17 times the risk of receptive VI. Another example is the change in condom risk-factor analysis. Although never offering $100 \%$ risk reduction, condoms worn correctly and consistently were considered the best available method of reducing infection.

Today it is acknowledged that having RAI with an HIV-infected partner using a condom carries a substantial transmission risk: 39 per 10,000 exposures (this 


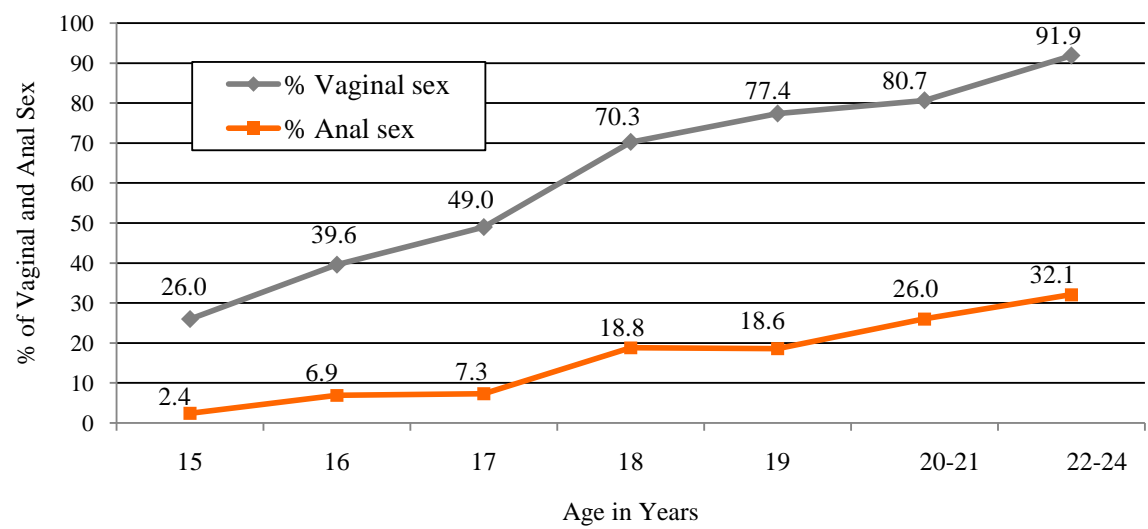

Figure 4. National Survey of Family Growth U.S. 2002. Heterosexual Vaginal and Anal Intercourse Reported by Young Women. Mosher WD, Chandra A, Jones J. Sexual behavior and selected health measures: men and women 15 - 44 years of age, United States, 2002. Adv Data. 2005 (362): 1-55.

risk is three and one-half times higher than having unprotected, insertive AI with an HIV-infected person: 11). With the addition of PrEP, the risk is further reduced: 3. However, the Table shows that the other risks of acquiring HIV infection via RAI are higher, and at times extreme, the range being 8 to 6840 . These risks are clearly much higher than insertive AI and receptive VI. Furthermore, among MSM, a recent long-term study revealed that consistent condom use during AI was low (16.4\%). Consequently, some of the factors above may in part explain the increase of HIV infection among young MSM from 2001-2009 and their persistent high HIV infection rates from 2010-2014.

That AI starts in adolescence and increases with age in both sexes reveals that this high-risk behavior is not unique to gender or lifestyle. Both sexes are at risk, especially in areas of high HIV prevalence. Moreover, health outcomes often are driven by risk behaviors established during adolescence. Preventing their initiation through education has lifelong health benefits [23]. Thus informing young people of the potentially harmful risks of RAI may likely result in a downward trend of new HIV infections and contribute to ending the epidemic.

In the past, the public was made aware that needle-sharing during IDU carried the highest risk for the transmission of HIV. The public responded and either decreased injecting drugs or decreased sharing needles (e.g., needle exchange programs). The result was that HIV infection attributed to IDU dropped by $80 \%$ [24] and continues to trend downward [12]. A similar strategy to alert the public that receptive anal intercourse (RAI) is an even greater risk than needle-sharing appears to be warranted.

\section{References}

[1] Centers for Disease Control and Prevention (2014) HIV Risk Behaviors. http://www.cdc.gov/hiv/policies/law/risk.html

[2] Patel, P., Borkowf, C.B., Brooks, J.T., Lasry, A., Lansky, A. and Mermin, J. (2014) Estimating Per-Act HIV Transmission Risk: A Systematic Review. AIDS, 28, 
1509-1519. https://doi.org/10.1097/QAD.0000000000000298

[3] Centers for Disease Control and Prevention (2006) Human Immunodeficiency Virus (HIV) Risk, Prevention, and Testing Behaviors-United States, National HIV Behavioral Surveillance System: Men Who Have Sex with Men, November 2003-April 2005. MMWR Surveillance Summaries, 55, 1-16.

[4] Finlayson, T.J., Le, B., Smith, A., et al. (2011) HIV Risk, Prevention, and Testing Behaviors among Men Who Have Sex with Men-National HIV Behavioral Surveillance System, 21 U.S. Cities, United States, 2008. MMWR Surveillance Summaries, 60, 1-34.

[5] Buchbinder, S.P., Vittinghoff, E., Heagerty, P.J., et al. (2005) Sexual Risk, Nitrite Inhalant Use, and Lack of Circumcision Associated with HIV Seroconversion in Men Who Have Sex with Men in the United States. Journal of Acquired Immune Deficiency Syndromes, 39, 82-89. https://doi.org/10.1097/01.qai.0000134740.41585.f4

[6] Mosher, W.D., Chandra, A. and Jones, J. (2005) Sexual Behavior and Selected Health Measures: Men and Women 15-44 Years of Age, United States, 2002. Advance Data, No. 362, 1-55.

[7] Copen, C.E., Chandra, A. and Febo-Vazquez. I. (2016) Sexual Behavior, Sexual Attraction, and Sexual Orientation Among Adults Aged 18-44 in the United States: Data From the 2011-2013 National Survey of Family Growth. National Health Statistics Report, No. 88, 1-14.

[8] Centers for Disease C, Prevention (2008) Trends in HIV/AIDS Diagnoses among Men Who Have Sex with Men-33 States, 2001-2006. MMWR Morbidity and Mortality Weekly Report, 57, 681-686.

[9] Centers for Disease Control and Prevention (2012) Diagnoses of HIV Infection and AIDS among Adolescents and Young Adults in the United States and 5 U.S. Dependent Areas, 2006-2009. HIV Surveillance Supplemental Report, 17, No. 2.

[10] Centers for Disease Control and Prevention (2016) Diagnoses of HIV Infection among Adolescents and Young Adults in the United States and 6 U.S. Dependent Areas, 2010-2014. HIV Surveillance Supplemental Report, 21, No. 3.

[11] The White House Office of National AIDS Policy (2015) National HIV/AIDS Strategy for the United States. The White House Office of National AIDS Policy, Washington DC.

[12] Centers for Disease Control and Prevention (2016) HIV Surveillance Report, 2015. U.S. Department of Health and Human Services, Atlanta, Vol. 27.

[13] Group, E.S. (1992) Comparison of Female to Male and Male to Female Transmission of HIV in 563 Stable Couples. European Study Group on Heterosexual Transmission of HIV. BMJ, 304, 809-813. https://doi.org/10.1136/bmj.304.6830.809

[14] Dasgupta, S., Oster, A.M., Li, J. and Hall, H.I. (2016) Disparities in Consistent Retention in HIV Care-11 States and the District of Columbia, 2011-2013. MMWR Morbidity and Mortality Weekly Report, 65, 77-82. https://doi.org/10.15585/mmwr.mm6504a2

[15] Stein, R., Song, W., Marano, M., Patel, H., Rao, S. and Morris, E. (2017) HIV Testing, Linkage to HIV Medical Care, and Interviews for Partner Services among Youths-61 Health Department Jurisdictions, United States, Puerto Rico, and the U.S. Virgin Islands, 2015. MMWR Morbidity and Mortality Weekly Report, 66, 629-635. https://doi.org/10.15585/mmwr.mm6624a2

[16] Centers for Disease Control and Prevention (2017) Monitoring Selected National HIV Prevention and Care Objectives by Using HIV Surveillance Data-United 
States and 6 Dependent Areas, 2015. HIV Surveillance Supplemental Report, 22, No. 2.

[17] Smith, D.K., Grohskopf, L.A., Black, R.J., et al. (2005) Antiretroviral Postexposure Prophylaxis after Sexual, Injection-Drug Use, or Other Nonoccupational Exposure to HIV in the United States: Recommendations from the U.S. Department of Health and Human Services. MMWR Morbidity and Mortality Weekly Report, 54, 1-20.

[18] Centers for Disease Control and Prevention (2016) Anal Sex and HIV Risk. http://www.cdc.gov/hiv/risk/analsex.html

[19] Smith, D.K., Herbst, J.H., Zhang, X. and Rose, C.E. (2015) Condom Effectiveness for HIV Prevention by Consistency of Use among Men Who Have Sex with Men in the United States. Journal of Acquired Immune Deficiency Syndromes, 68, 337-344. https://doi.org/10.1097/QAI.0000000000000461

[20] Centers for Disease Control and Prevention (2016) HIV Risk Reduction Tool (Beta Version). https://wwwn.cdc.gov/hivrisk/

[21] Fleming, D.T. and Wasserheit, J.N. (1999) From Epidemiological Synergy to Public Health Policy and Practice: The Contribution of Other Sexually Transmitted Diseases to Sexual Transmission of HIV Infection. Sexually Transmitted Infections, 75, 3-17. https://doi.org/10.1136/sti.75.1.3

[22] Centers for Disease Control and Prevention (1998) HIV Prevention through Early Detection and Treatment of Other Sexually Transmitted Diseases-United States. Recommendations of the Advisory Committee for HIV and STD Prevention. MMWR Morbidity and Mortality Weekly Report, 47, 1-24.

[23] Banspach, S., Zaza, S., Dittus, P., Michael, S., Brindis, C.D. and Thorpe, P. (2016) CDC Grand Rounds: Adolescence-Preparing for Lifelong Health and Wellness. MMWR Morbidity and Mortality Weekly Report, 65, 759-762. https://doi.org/10.15585/mmwr.mm6530a2

[24] Centers for Disease C, Prevention (2009) HIV Infection among Injection-Drug Users-34 States, 2004-2007. MMWR Morbidity and Mortality Weekly Report, 58, 1291-1295. 